\begin{tabular}{ccc}
\hline & Middle East Journal of Science \\
$\begin{array}{c}\text { INTERNEG } \\
\text { ENGINEERING, } \\
\text { SCIENCE AND } \\
\text { EDUCATION GROUP }\end{array}$ & e-ISSN:2618-6136 & DOI: $10.23884 /$ mejs.2019.5.2.03
\end{tabular}

Research Article

\title{
EFFECT OF ELECTROMAGNETIC FIELD ORIGINATING FROM HIGH VOLTAGE LINES ON MALONDIALDEHYDE LEVEL
}

\author{
"Mehmet Cihan YAVAŞ iD \\ Department of Biophysics, Faculty of Medicine, Kırşehir Ahi Evran University, Kırşehir, Turkey \\ *Corresponding author: mcihanyavas@gmail.com
}

\begin{abstract}
Our study aimed to investigate the effect of electromagnetic fields originating from high voltage lines on serum malondialdehyde level of male rats wistar albino. A total of 32 rats were randomly assigned to study in 4 groups. Groups were; Group 1: high voltage, Group 2: high voltage+ganoderma l., Group 3: high voltage+melatonin, Group 4: control. Experimental groups were exposed to high voltage for 8 hours daily for 52 days. The electric field and the magnetic field were measured. Ganoderma was administered $20 \mathrm{mg} / \mathrm{kg} /$ day as a gavage and melatonin intraperitoneally as $10 \mathrm{mg} / \mathrm{kg} /$ day. In the study, the malondialdehyde (MDA) levels of the experimental and control groups were compared. There was no significant difference between the groups. According to the control group, it was found that the MDA level of the high voltage group increased, while the Ganoderma and Melatonin groups had a small decrease at the MDA level. These results show that electromagnetic fields originating from high voltage increase the MDA serum level, which is found to decrease in the presence of ganoderma and melatonin.
\end{abstract}

Keywords: Malondialdehyde, High voltage, Melatonin, Ganoderma, Electromagnetic field

Received: July 5, $2019 \quad$ Accepted: October 10, 2019

\section{Introduction}

With the development of technology, the use of electric vehicles has become increasingly widespread, so pollution arising from very low-frequency electromagnetic fields is gradually increasing [1]. Electromagnetic fields related to the increase in mobile phones, televisions, computers, electrical appliances and occupational exposures used in the home and business environment and negative effects on the human body have caused worries and problems. Most of the studies have shown that these areas are a negative effect, but some studies report that these areas have a positive (therapeutic) orientation [2]. There are studies of how long-term exposures of very low-frequency electromagnetic fields can reason oxidative DNA harm [3]. The main source of malondialdehyde is lipid peroxidation of polyunsaturated fatty acids. In most studies, malondialdehyde is used because it is a natural marker for oxidative stress. Several malondialdehyde methods have been developed since 1960 to determine oxidative stress in vivo and in vitro studies [4]. The polysaccharides obtained from Ganoderma lucidum showed free radical cleansing, anti-angiogenic, anti-tumor and immunosuppressive properties [5]. Melatonin has the ability to neutralize oxidative stress-related tissue damage and free radicals [6].

\section{Materials and Methods}

This work was conducted at the Dicle University Health Sciences Research Center. Permission was obtained from the Ethics Committee of the University of Dicle Animal Experiments (DUHADEK: 2013/13) and the study continued according to the standards set out in the Helsinki Declaration. 


\subsection{Animals and experimental protocol}

A total of 32 rats (weighing 320,45 $\pm 6,84,4-5$ months) were randomly assigned to study in 4 groups. Groups were; Group 1: HV, Group 2: HV+GI, Group 3: HV+MEL, Group 4: Control. Experimental groups were exposed to high stress for 8 hours daily for 52 days. All animals were kept in suitable environmental conditions (constant temperature of $23 \pm 1{ }^{\circ} \mathrm{C}$ and $45-55 \%$ humidity of the air), 12 hours of the night and 12 hours of light daylight, respectively, normal food (ad-libitum) and water were always available in the animals' cage. To create electromagnetic fields, two transformers that produced 10 kiloVolt $(10,000 \mathrm{~V})$ of high voltage were used. For the first transformer, the input was 220 Volt, and the output was 10 kiloVolt. For the second transformer, the input was 10 kiloVolt, and the output was 220 Volt and 5,000 Volt Amper. The electric field $(80,3 \mathrm{~V} / \mathrm{m})$ and the magnetic field $(2,48$ $\mu \mathrm{T})$ were measured. Ganoderma (Gano Excel, Industries Sdn. Bhd., Kedah, Malaysia) extracts were prepared with distilled water with appropriate standards. Melatonin (Merck KGaA, Germany) was prepared according to the weights of rats and then dissolved with pure ethanol and dissolved in distilled water at appropriate ratios. Ganoderma was given as $20 \mathrm{mg} / \mathrm{kg} /$ day as a gavage and melatonin were administered intraperitoneally as $10 \mathrm{mg} / \mathrm{kg} / \mathrm{day}$. Electromagnetic field measurement The Spectran NF5035 (AARONIA AG, Strickscheid, Germany), the instrument was taken in 6-minute measurements as determined by ICNIRP.

\subsection{Blood collection and detection of MDA}

After the study, rats were anesthetized by administering ketamine-xylazine at appropriate ratios, blood samples taken from the sacrificed rats were studied. Blood samples were immediately centrifuged at $500 \mathrm{rpm}$ for 5 minutes to collect serum for study. Assay protocol MDA-TBA (Thiobarbituric acid) (Product NWK-MDA01, Northwest, Life Science Specialties, Vancouver); Add $10 \mu \mathrm{L}$ BHT Reagent to microcentrifuge vial. Add $250 \mu \mathrm{L}$ calibrator or sample to the vial. Add $250 \mu \mathrm{L}$ Acid Reagent to the vial. Add $250 \mu \mathrm{L}$ TBA Reagent to the vial. To make a homogenous structure. Vortex V1 Plus vigorously (5-count) (Boeco, Germany). Then, Incubate 60 minutes at $60{ }^{\circ} \mathrm{C}$ (Nuve bath, Germany). Centrifuge at 10,000 rpm for 2-3 minutes (M-240R, Boeco, Germany). Transfer reaction mixture to the cuvette. MDA serum samples were read on a UV-VIS spectrophotometer at $515 \mathrm{~nm}$. (UV-1208, Shimadzu, Japan) Then Perform $3^{\text {rd }}$ derivative analysis. The results were taken as $\mu \mathrm{L}$ [7].

\subsection{Statistical analysis}

The Kolmogorov-Smirnov test was used to assess whether the data in our study were in the normal distribution. Our test results show normal distribution. Since the number of observation is small, we used the Kruskal-Wallis test and Mann Whitney U test to evaluate the data statistically. $p<0.05$ was considered statistically significant. Explanatory statistical evaluations such as mean, standard deviation, minimum, maximum, median and $\mathrm{p}$ significance were determined. For these analyses, the SPSS 21. 0 (IBM SPSS Statistics for Windows, version 21.0, Armonk, NY: IBM Corp., USA) package program was used.

\section{Results}

In the study, the MDA levels of the experimental and control groups were compared. There was no significant difference between the groups $(>0.05)$. According to the control group, it was found that 
the MDA level of the high voltage group increased, while the Ganoderma and Melatonin groups had a small decrease at the MDA level. MDA values of all groups are shown in Table 1. In Table 2, binary group comparisons were made and it was found that there was a significant change between group 1 and group 2 and between group 1 and group 3. The addition of melatonin and Ganoderma when compared with the high voltage group gave a statistically significant result $(<0.05)$. In Figure 1, MDA concentration levels of all groups are given graphically together.

Table 1. MDA levels of all groups

\begin{tabular}{|c|c|c|c|c|}
\hline Groups / 52 day & Mean+ SD & Median & Min & Max \\
\hline $\begin{array}{ll}\text { Group 1 } & \text { HV }\end{array}$ & $12,34 \pm 1,95$ & 11,90 & 9,43 & 15,52 \\
\hline Group 2 & $9,27 \pm 2,46$ & 10,25 & 5,27 & 11,55 \\
\hline $\begin{array}{ll}\text { Group } 3 & \text { HV+MEL }\end{array}$ & $9,09 \pm 3,65$ & 9,37 & 2,86 & 13,54 \\
\hline Group 4 Control & $10,90 \pm 1,85$ & 11,37 & 7,74 & 12,67 \\
\hline \multirow{2}{*}{$\mathrm{P}$} & \multicolumn{4}{|c|}{ Chi-square:6,981 } \\
\hline & $\mathrm{P}>0,05$ & \multicolumn{3}{|l|}{ df: 3} \\
\hline
\end{tabular}

Values are given as mean \pm standard deviation (SD), Median, Minimum (Min) and Maximum (Max). $\mathrm{P}>0.05$ was not found significant with kruskal-wallis anova test.

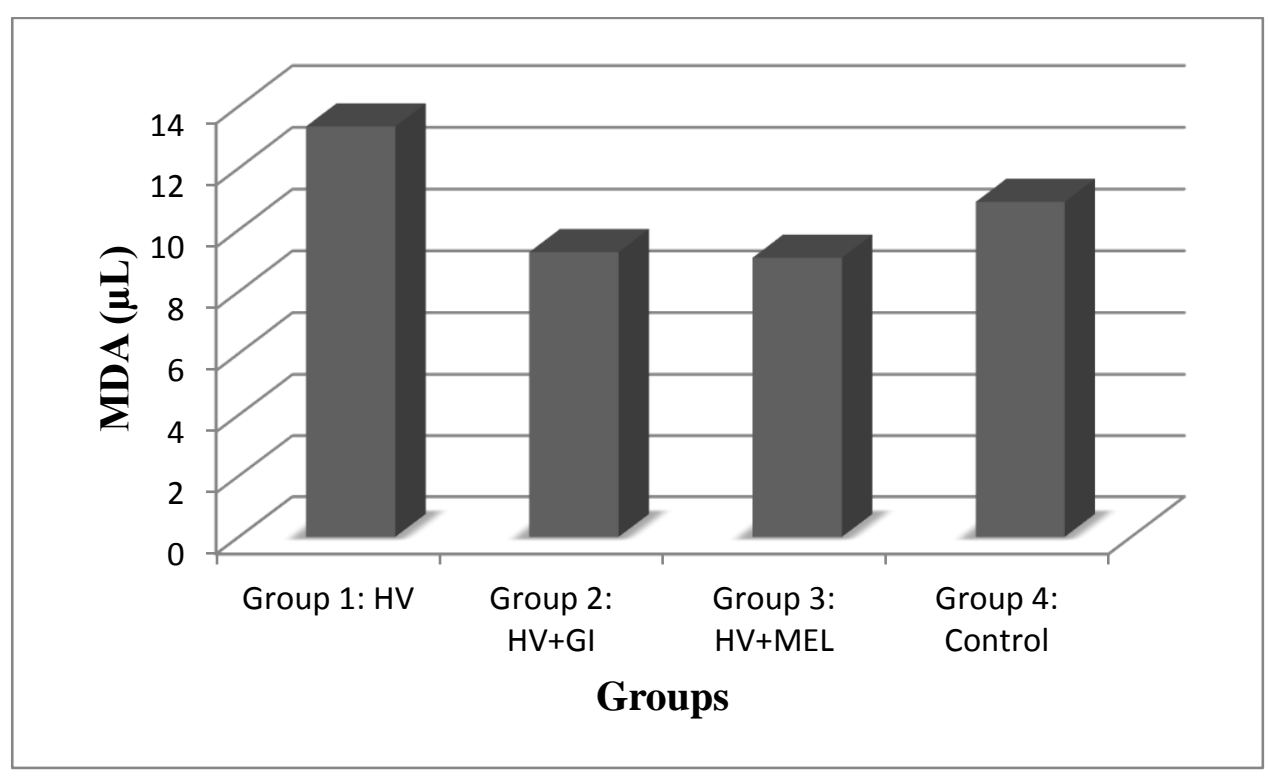

Figure 1. Graphical representation of MDA serum concentrations of rats. MEL: melatonin, HV: high voltage, GI: Ganoderma lucidum 
Table 2. Binary comparisons of all groups

\begin{tabular}{|l|ll|}
\hline Groups & P & \\
\hline Group 1-Group 2 & $\mathrm{P}: 0,015$ & $\mathrm{P}<0,05^{*}$ \\
\hline Group 1-Group 3 & $\mathrm{P}: 0,046$ & $\mathrm{P}<0,05^{*}$ \\
\hline Group 1-Group 4 & $\mathrm{P}: 0,343$ & $\mathrm{P}>0,05$ \\
\hline Group 2-Group 3 & $\mathrm{P}: 0,752$ & $\mathrm{P}>0,05$ \\
\hline Group 2-Group 4 & $\mathrm{P}: 0,172$ & $\mathrm{P}>0,05$ \\
\hline Group 3-Group 4 & $\mathrm{P}: 0,293 \quad \mathrm{P}>0,05$ \\
\hline
\end{tabular}

Binary group comparisons were made by Mann Whitney u test.

$* \mathrm{P}<0.05$ was statistically significant in the binary comparisons.

\section{Discussion}

In a study conducted by Meral et al. [8], $900 \mathrm{MHz}$ electromagnetic field was found to produce oxidative stress on guinea pigs brain tissue and blood parameters and it was found that the experimental group increased statistically compared to MDA level control group $(\mathrm{p}<0.05)$. In the groups exposed to $50 \mathrm{~Hz}$ and 80 Gauss electromagnetic fields, the MDA level of the experimental group was found to increase and the total antioxidant level decreased [9]. Magnetic field (50 Hz MF of $1.5 \mathrm{mT}$ ) continuous (4 h/day) and intermittent (two h/2h) exposure were found to alter antioxidant status in different tissues and significantly increase malondialdehyde level of intermittent exposures [10]. In the study conducted by Türközer and his colleagues on the Guinea pig, it was seen that the average of MDA increased with the increase of electric field compared to the sham group [11]. Çelik and colleagues investigated the effect of hairdryers device (ELF-EMA) on serum MDA used in hairdressing salons. From the serum, the oxidative effect of the ELF electromagnetic field was reported to be caused by an increase in the MDA level of the experimental group [12]. Similarly, Kula and his colleagues investigated the effect of electromagnetic fields on workers in the steel industry for 3-10 years. In the study data, it is seen that there is an increase in the plasma MDA level with the increase of the study period according to the control group [13]. In our study, it was observed that the MDA level was higher in the high voltage group than in the control group. In our study, MDA level in high voltage+melatonin and high voltage+ganoderma groups were found to be closer to the control group and decrease with respect to the high voltage group. In our study, we have determined that high-voltage electromagnetic exposures can change MDA level. Our work is consistent with the literature.

Melatonin, secreted from the pineal gland, has so far proved to be a good antioxidant. At the same time, many studies have reported that melatonin is protective against oxidative stress $[14,18,19$, 20]. Researchers investigated the effect of melatonin on lipid peroxidation during radiotherapy in female rats. Blood and tissue malondialdehyde level of radiotherapy groups were found higher than the control group. MDA levels in melatonin-treated groups showed a decrease compared to the radiotherapy group. These results report that melatonin reduces MDA levels [15]. Liver-tumorled mices were exposed to 6 Gy gamma radiation. They reported that ganoderma given to mices may have an anti-tumor effect [16]. Researchers used ganoderma extracts that have an antioxidant effect on cardiac toxicity. The study results reported that ganoderma may have cardioprotective effects. Thus, it has been found that Ganoderma lucidum inhibits lipid peroxidation and significantly reduces malondialdehyde (MDA) formation [17]. Statistical binary comparisons of our study showed a significant difference between Group 1 (high voltage) - Group 2 (voltage + ganoderma) and Group 1 (high voltage) - Group 3 (high 
voltage + melatonin $)(\mathrm{p}<0.05)$. This statistical difference suggests that malondialdehyde levels may change when added with ganoderma and melatonin, and the harmful effects caused by oxidative stress can be repaired.

\section{Conclusion}

In our study, ELF-EMA exposure is considered to increase lipid peroxidation and oxidative stress. This effect appears to originate from the electromagnetic field originating from high voltage. Thus, after the addition of Ganoderma and Melatonin gave to the groups, the increased level of MDA was partially reduced, indicating that melatonin and ganoderma had a protective effect.

\section{Acknowledgments}

This work was presented as an oral presentation at the 30th Annual Biophysics Congress (International) OP-20, October 10-13, 2018, Bodrum- Turkey.

\section{Funding information}

No financial support was received in the study.

\section{Disclosure statement}

The author states that there is no conflict of interest

\section{References}

[1] Seifirad, S., Farzampour, S., Nourbakhsh, M., et al. "Effects of extremely low-frequency electromagnetic fields on paraoxonase serum activity and lipid peroxidation metabolites in rat", $J$ Diabetes Metab Disord, 13, 85-94, 2014.

[2] Kocaman, A., Altun, G., A.Kaplan, A., et al. "Genotoxic and carcinogenic effects of non-ionizing electromagnetic field”, Environ Res, 163, 71-79, 2018.

[3] Yokuş, B., Ülker, Çakır D., Akdağ, MZ., et al. "Oxidative DNA damage in rats exposed to extremely low-frequency electromagnetic fields", Free Radic Res, 39(3), 317-323, 2005.

[4] Del Rio, D., J Stewart, A., Pellegrini, NA., "Review of recent studies on malondialdehyde as toxic molecule and biological marker of oxidative stress", Nutr Metab Cardiovasc Dis, 15, 316-328, 2005.

[5] Smina, TP., De, Strayo., Devasagayam, TPA., et al. "Ganoderma lucidum total triterpenes prevent radiation-induced DNA damage and apoptosis in splenic lymphocytes in vitro", Mutat Res, 726, 188194, 2011.

[6] Espino, J., Pariente, JA., Rodriguez, AB., "Oxidative stress and immunosenescence: therapeutic effects of melatonin”, Oxid Med Cell Longev, 2012, 9 Pages, 2012.

[7] Jentzsch, AM., Bachmann, H., Fürtst, P., K Biesalski, H., "Improved analysis of malondialdehyde in human body fluids", Free Rad Biol Med, 20, 251-256, 1996.

[8] Meral, İ., Mert, H., Mert, N., et al. "Effects of 900-MHz electromagnetic field emitted from cellular phone on brain oxidative stress and some vitamin levels of guinea pigs", Brain Res, 1169, 120-124, 2007.

[9] Hajhosseini, L., Khaki, A., Merat, E., et al. "Effect of rosmarinic acid on sertoi cells apoptosis and serum antioxidant levels in rats after exposure to electromagnetic fields", Afr J Tradit Complement Altern Med, 10(6), 477-480, 2013. 
[10] Çoşkun, Ş., Balabanl1, B., Canseven, A., et al. "Effects of continuous and intermittent magnetic fields on oxidative parameters in vivo", Neurochem Res, 34, 238-243, 2009.

[11] Türközer, Z., Güler, G., Seyhan, N., "Effects of exposure to $50 \mathrm{~Hz}$ electric field at different strengths on oxidative stress and antioxidant enzyme activities in the brain tissue of guinea pigs", Int $J$ Radiat Biol, 84(7), 581-590, 2008,

[12] Çelik, MS., Akpolat, V., Işık, B., et al. "The effect of low-frequency electromagnetic field (ELFEMF) on serum paraoxanase (PON1) and malondialdehyde (MDA) levels", Int Arc Med Res, 6(1), 1-4, 2014.

[13] Kula, B., Sobczak, A., Kuska, R., "Effect of electromagnetic field on free-radical processes in steelworkers. part I: magnetic field influence on the antioxidant activity in red blood cells and plasma", J. Occup Health, 44, 226-229, 2002.

[14] Galano, A., Tan, DX., J Reiter, R., "Melatonin as a natural ally against oxidative stress: a physicochemical examination", J Pineal Res, 51, 1-16, 2011.

[15] Kaya, H., Delibas, N., Serteser, M., et al. "The effect of melatonin on lipid peroxidation during radiotherapy in female rats", Strahlenther Onkol, 175, 285-8, 1999,

[16] Ibrahim, SI., El-Kabany, H. "Effect of Ganoderma lucidum (g. lucidum) on the liver of mice bearing ehrlich solid tumor (EST) and exposed to $\gamma$-radiation”, J Rad Res Appl Sci, 6(1), 175-196, 2013.

[17] Wong, KL., Chao, HH., Chan, P., et al. "Antioxidant activity of Ganoderma lucidum in acute ethanol-induced heart toxicity", Phytother Res, 18, 1024-1026, 2004.

[18] Doğan, MS., Yavaş, MC., Yavuz, Y., Erdoğan, S., Yener, İ., Şimşek, İ., et al. "Effect of electromagnetic fields and antioxidants on trace element content in rat teeth", "Drug Des Devel Ther, 11, 1393-1398, 2017.

[19] Doğan, MS., Yavaş, MC., Günay, A., Yavuz, İ., Deveci, E., Akkuş, Z., et al. “The protective effect of melatonin and ganoderma lucidum against the negative effects of extremely low frequency electricity and magnetic fields on pulp structure in rat teeth", Biotechnol Biotechnol Equip, 31 (5), 979-988, 2017. [20] Tanık, A, Yavaş, MC., "The protective effect of melatonin and ganoderma lucidum aganist the negative effect of extremely low-frequency electromagnetic fields (EMF) on alveolar bone of rats", Anal Quant Cytol. 41(2), 55-66, 2019. 